\title{
The relationships between intra-abdominal echogenicity, cardiometabolic risk factors and physical performance in obese children
}

\author{
Ji Won Yoo ${ }^{\mathrm{a}}$, Nam-Gi Lee ${ }^{\mathrm{b}}$, Hee-Jung Kim ${ }^{\mathrm{c}}$, Hyo-Min Cho ${ }^{\mathrm{c}}$ and Joshua H. You ${ }^{\mathrm{a}, *}$ \\ ${ }^{a}$ Department of Physical Therapy, Yonsei University, Wonju City, Kangwon-do, Republic of South \\ Korea \\ ${ }^{b}$ Department of Physical Therapy, College of Health and Welfare, Woosong University, Republic of \\ South Korea \\ ${ }^{c}$ Department of Radiological Science Yonnsei University, Wonju City, Kangwon-do, Republic of South \\ Korea
}

\begin{abstract}
While the abdominal adipose tissue has been identified as an important pathomarker for the cardiometabolic syndrome in adults, the relationships between the cardiometabolic risk factors and abdominal adipose morphology or physical performance levels have not been examined in children with obesity. Therefore, the specific aim of this study was to investigate the relationships between risk factors (BMI and physical activity levels and abdominal fat layers including subcutaneous, intra-abdominal preperitoneal and mesenteric fat thickness in children with obesity. 30 children with obesity (mean $\pm \mathrm{SD}=10.0 \pm 4.5 \mathrm{yrs} ; 9$ girls; BMI > 20) underwent physical performance (curl-ups, sit and reach, push-ups, and a 400$\mathrm{m}$ run), ultrasound measurement of thickness of fat composition of the abdomen, blood pressure, oxygen consumption. Pearson correlation analysis showed significant correlations, ranging from -0.523- 0.898 between the intra-abdominal adipose tissue thickness, cardiometabolic risk factors (BMI, blood pressure, heart rate), and the curl-up physical performance test. In conclusion, the present study provides a compelling evidence that the intra-abdominal adipose tissue morphological characteristics were associated with BMI, physical performance, and most importantly cardiometabolic risk factors (blood pressure and heart rate), which eventually contribute to the development of cardiometabolic syndrome in adulthood.
\end{abstract}

Keywords: Ultrasound, BMI, child obesity, cardiometabolic syndrome

\section{Introduction}

Obesity in childhood is a common, leading cause of chronic cardio-metabolic diseases in adults, including diabetes, hypertension, and dyslipidemia [1,2]. Epidemical research statistics have revealed that approximate 16-30 percent of children (over 9 million) between age of 6-19 years old are reported to be overweight [1,2]. Moreover, over the past three decades the childhood obesity rate has more than doubled for preschool children aged 2-5 years and adolescents aged 12-19 years, and tripled for

\footnotetext{
${ }^{*}$ Corresponding author: Joshua (Sung) H. You, Department of Physical Therapy, Yonsei University, Wonju City, Kangwon-do, Republic of South Korea. Tel.: 033-760-2476; Fax: 033-760-2496; E-mail: neurorehab@yonsei.ac.kr.
}

0959-2989/14/\$27.50 @ 2014 - IOS Press and the authors. 
children aged 6-11 years [1,3]. Child obesity is an important biomarker for cardio-metabolic syndrome in adulthood including central obesity, diabetes, high blood pressure, prothrombotic state and dyslipidemia $[1,2,4]$. Understanding metabolic and morphological courses in childhood obesity has therefore crucial clinical ramifications for the effective prevention and management of cardiometabolic syndrome in adulthood.

Childhood obesity has been assessed by BMI, physical activity, and diagnostic imaging methods including ultrasound (US), computed tomography (CT), and MRI. While BMI is easy and simple to administer, but does not provide morphological changes associated with obesity. Diagnostic imaging techniques have been used to provide precise quantitative information for measuring body fat distribution, but the disadvantages are costly, time-consuming, and somewhat hazardous because of ionizing radiation exposure for $\mathrm{CT}$, and use limited to a research setting [5-7]. In contrast, a real-time ultrasonography (US) is relatively inexpensive and portable and provides accurate morphological estimation of body fat composition [5,7,8]. Recently, US imaging has been used to accurately determine subcutaneous and intra-abdominal; preperitoneal and visceral fat thickness.

Visceral and mesenteric fat are a specific type of visceral adipose tissue which is drained by the portal circulation, and has been associated with different metabolic patterns compared with other types of adipose tissues such as subcutaneous and peritoneal fat [9-11]. The ultrasonic measurement of thickness of mesenteric fat, which represents a more specific and accurate deposit of visceral fat, showed a good correlation with cardiovascular risk factors in healthy persons $[2,9,12,13]$. However, there is a dearth of evidence that examined the relationship between fat composition, BMI, blood pressure, oxygen consumption level, and physical activity including curl-up, sit and reach, and pushup in children with obesity.

Therefore, the specific aim of this study is to investigate the relationships between risk factors (BMI and physical activity levels) and abdominal fat layers including subcutaneous, intra-abdominal preperitoneal and mesenteric fat thickness as measured on US scan in children with obesity. We hypothesized that the relationships between the cardiometabolic risk factors (BMI, blood pressure) and abdominal adipose morphology, as well as the cardiometabolic risk factors and physical performance levels, would exist in children with obesity.

\section{Materials and methods}

\subsection{Subjects}

A convenience sample of 30 children (mean $\pm \mathrm{SD}=10.0 \pm 4.5 \mathrm{yrs} ; 9$ girls) with obesity were recruited from multiple elementary schools as a part of FITKIDS program. All parents of the children candidate provided informed consent form that was approved by Yonsei University. Inclusion criteria entailed: (1) age ranging from 5-15 years old; (2) $>20$ in the body mass index scale; (3) healthy. Exclusion criteria included those children with congenital heart problems, diabetes, and any other medical problems that could affect physical performance tests. The baseline of health and physical performance was prescreened by a demographic and health screening questionnaire. 


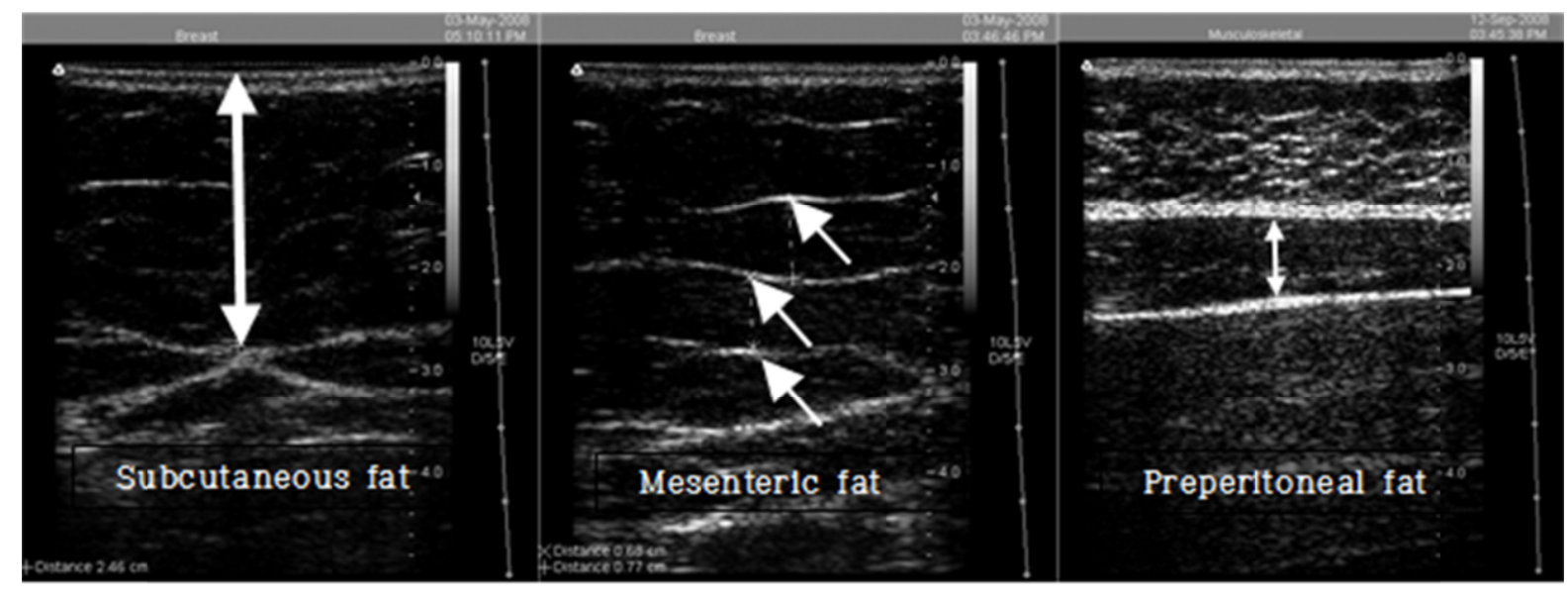

Fig. 1. Ultrasound measurement of thickness of intra-abdominal echogenicity and fat composition of the abdomen in children with obesity.

\subsection{Physical performance test}

A standardized physical fitness assessment was used to examine physical performance and fitness level of obese children. The test included curl-ups, sit and reach, push-ups, and a 400-m run test [3,14].

\subsection{Ultrasonic measurement of body fat composition}

A real-time ultrasound measurement (SonoAce X4) was used to measure the thickness of fat composition of the abdomen for all children in experiment, with a 5-9 $\mathrm{MHz}$ curvilinear transducer (Medison Ltd, Seoul, Korea). Each child was comfortably positioned in a relaxed hook-lying position $[3,7]$ with one or two pillows under the knees to eliminate excessive lumbar lordosis. Ultrasound gel (AQUASONIC ${ }^{\circledR} 100$ ) was then applied to the transducer head. An exploratory US imaging with emphasis in the region of the interest (ROI) such as para-umbilical area was performed to identify the mesenteric leaves (Figure 1). The transducer head was then maneuvered to acquire the clearest image of the mesenteric leaves. The mesenteric fat (MF) leaves was anatomically defined a series of the adipose tissue with highly reflecting peritoneal surfaces differentiated from each other by specular echoes $[5,15]$. Preperitoneal fat (PF) thickness (Figure 1) was measured by orienting the transducer longitudinally whereas the subcutaneous fat (SF) thickness (Figure 1) was measured by orienting it transversely, which was perpendicular to the ROI of the para-umbilical skin located between the xiphoid process and umbilicus $[5,13,15]$. Once the sharpest images of the mesenteric leaves were visualized on the monitor, the three maximal thickest mesenteric leaves were determined by vertically drawing the mark line with a caliper and stored for the further statistical analysis.

\subsection{Statistics}

Standard statistical analysis was made for data, including computations of means and standard deviations, and Pearson correlation. The SPSS version 12.0 for Windows was used to compute a statistical significance at $P<0.05$. 
Table 1

Clinical characteristics and measurement of abdominal adipose tissues

\begin{tabular}{ll}
\hline Characteristics & Mean \pm SD \\
\hline Number & 30 \\
Age & $10.71 \pm 1.58$ \\
Gender (male/female) & $12 / 8$ \\
Height $(\mathrm{cm})$ & $141.68 \pm 9.82$ \\
Wright(kg) & $50.85 \pm 10.72$ \\
400m run(sec) & $180.04 \pm 43.17$ \\
HR (before) & $94.61 \pm 14.06$ \\
HR (after) & $149.75 \pm 25.10$ \\
O2 (before) & $98.86 \pm 0.93$ \\
O2 (after) & $98.21 \pm 1.23$ \\
BP (before) (Systolic/diastolic) & $117.43 \pm 11.28 / 72.04 \pm 7.46$ \\
BP (after) (Systolic/diastolic) & $150.36 \pm 15.03 / 74.21 \pm 14.79$ \\
BMI(kg/m2) & $24.89 \pm 2.26$ \\
Curl-Ups(EA) & $18.71 \pm 10.36$ \\
Sit and Reach(cm) & $4.98 \pm 7.77$ \\
Push-Ups(EA) & $10.82 \pm 7.56$ \\
Mesenteric fat(MF) & $0.52 \pm 0.19$ \\
Preperitoneal fat(PF) & $0.75 \pm 0.30$ \\
Subcutaneous fat(SF) & $2.07 \pm 0367$ \\
\hline
\end{tabular}

\section{Results}

\subsection{Anthropometrical and demographic data}

Table 1 presents the anthropometrical and clinical characteristics of the children. The mean BMI was $24.89 \pm 2.26$, suggesting a mild to moderate obesity (Figure 1).

\subsection{Intra-abdominal adipose tissue thickness data}

Table 2 represents a comparison of the intra-abdominal adipose tissue thickness data in the MF, PF, and SF. Pearson or Intraclass correlation coefficient (ICC) analysis showed significant correlations, $r$ $=0.48$ between MF and PF, and $r=0.76$ between MF and SF, respectively.

\subsection{Reliability data}

Table 2 represents correlation coefficient data of various obesity index, intra-abdominal adipose tissue thickness, and cardiometabolic risk factors, which ranged from $-0.523-0.898$. 
Table 2

Correlation coefficients of various obesity indexes with cardiovascular risk factors

\begin{tabular}{|c|c|c|c|c|c|c|c|c|c|}
\hline & MF & $\mathrm{PF}$ & SF & BMI & Weight & Height & $\begin{array}{l}\text { Curl- } \\
\text { Ups }\end{array}$ & $\begin{array}{l}\text { Push- } \\
\text { Ups }\end{array}$ & $\begin{array}{l}\text { Sit and } \\
\text { Reach }\end{array}$ \\
\hline $\mathrm{MF}$ & 1 & & & & & & & & \\
\hline $\mathrm{PF}$ & $.477 * *$ & 1 & & & & & & & \\
\hline SF & $.755^{* * *}$ & $.525^{* *}$ & 1 & & & & & & \\
\hline BMI & $.763 * * *$ & $.421^{*}$ & $.787 * * *$ & 1 & & & & & \\
\hline Weight & $.752 * * *$ & $.523 * *$ & $.802 * * *$ & $.817 * * *$ & 1 & & & & \\
\hline Height & $.528 * *$ & $.533 * *$ & $.613 * * *$ & $.518^{* *}$ & $.898 * * *$ & 1 & & & \\
\hline Curl-Ups & $-.467^{*}$ & -.083 & $-.368^{*}$ & $-.484 * *$ & -.275 & -.001 & 1 & & \\
\hline Push-Ups & -.026 & -.122 & -.132 & -.104 & -.033 & .124 & $.470 *$ & 1 & \\
\hline $\begin{array}{l}\text { Sit and } \\
\text { Reach }\end{array}$ & $-.433^{*}$ & $-.380^{*}$ & $-.476^{*}$ & $-.505^{* *}$ & $-.527 * *$ & $-.450 *$ & .091 & .135 & 1 \\
\hline $\begin{array}{l}\text { Systolic } \\
\text { Pressure }\end{array}$ & $.540 * *$ & .191 & $.502 * *$ & $.587 * *$ & $.446^{*}$ & .198 & -.289 & -.223 & -.285 \\
\hline $\begin{array}{l}\text { Diastolic } \\
\text { Pressure }\end{array}$ & $.464^{*}$ & $.546^{* *}$ & $.573^{* *}$ & $.489 * *$ & $.437^{*}$ & .318 & -.181 & -.271 & $-.523^{* *}$ \\
\hline $\begin{array}{l}\text { Heart } \\
\text { Rate }\end{array}$ & -.145 & $-.420^{*}$ & -.242 & -.122 & -.339 & $-.454^{*}$ & -.047 & -.015 & -.096 \\
\hline
\end{tabular}

Note: MF represents Mesenteric fat, PF represents Preperitoneal fat, SF represents Subcutaneous fat.

$* P<0.05 ; * * P<0.01 ; * * * P<0.001$

\section{Discussion}

The present study is the first clinical evidence highlighting the relationship between the various obesity index, intra-abdominal adipose tissue thickness, and cardiometabolic risk factors in children with obesity. As anticipated, significant correlations between the BMI, intra-abdominal adipose tissue thickness, cardiometabolic risk factors (blood pressure and heart rate), and physical performance level were found. Most important, the ultrasound measurement of the intra-abdominal adipose tissue provides an important diagnostic information about the pathomarkers associated with cardiometabolic risk factors in children with obesity, which can be used for accurate evaluation and effective intervention of predisposed children with the cardiometabolic syndrome. Because in the current literature there was no previous data available in children with obesity, it was practically difficult to compare our present results.

Correlation analysis showed a strong relationship existed between BMI and the intra-abdominal adipose tissue thickness, particularly in the SF (0.79) and MF (0.76), indicating that abdominal fat metabolism is closely linked with abdominal morphology $[5,11,13,16-18]$. This finding was consistent with other study showing a positive correlation between visceral fat and BMI in adults [13,16-18]. Recently growing evidence suggests that abdominal fat metabolism is an important biomarker of insulin resistance Type I diabetes $[11,12,17,18]$. In adults, MF is believed to be sensitive to the catecholamine's effect, leading to increased FFA and insulin resistance by decreasing peripheral glucose uptake $[12,13,16-18]$. A moderate relationship was observed between all abdominal adipose tissues (PF, MF, SF) BMI and diastolic blood pressure whereas PF and MF were correlated with BP. This finding supports the fact that the visceral adipocytes, which are the progenies of the abdominal 
fat deposits play a role in releasing cytokines and vascoactive peptides (i.e., interleukin-6, angiotensin II), which can increase risk of cardiometabolic symdrome [4,5,17,18]. A strong relationship was found between BMI or MF and physical performance (i.e., curl-up), indicating that relative lack of abdominal muscle strength associated with muscle size is inversely associated with MF or BMI. This data corroborates previous finding showing that abdominal physical performance level is crucial for mesenteric fat deposit.

In smmary, these results suggest that the intra-abdominal adipose tissue morphological characteristics were associated with BMI, physical performance, and most importantly, cardiometabolic risk factors (blood pressure and heart rate), which eventually contribute to the development of cardiometabolic syndrome in adulthood. The childhood obesity is closely linked with other cardio-metabolic risk factors including hypertension, type 2 diabetes, and adiponectin adulthood $[1,2,18]$. However, the present study did not examine this relationship. Nevertheless, the present study has important clinical implications that child hood obesity is strongly linked with the intra-abdominal adipose tissue, physical performance, and most importantly cardio-metabolic risk factors (blood pressure and heart rate), which can be used to predict and/or to take preventive measures against diabetes or other cardio-metabolic syndromes in adulthood. Future longitudinal studies should investigate the cardio-metabolic risk factors including hypertension and diabetes in adulthood in order to test a possible hypothesis for the pathogenesis for the cardio-metabolic syndrome. Moreover, the ultrasound imaging measurement is an appropriate diagnostic tool to precisely ascertain the underlying pathogensis of the cardiometabolic syndrome in children with obesity.

\section{Acknowledgement}

Both Yoo and Lee have equally contributed to this project. This study was supported by the Wonju city health and welfare department.

\section{References}

[1] G. Rao, Childhood obesity: Highlights of AMA Expert Committee recommendations, American Family Physician 78 (2008), 56-63.

[2] P.W. Franks, R.L. Hanson, W.C. Knowler, M.L. Sievers, P.H. Bennett and H.C. Looker, Childhood obesity, other cardiovascular risk factors, and premature death, New England Journal of Medicine 362 (2010), 485-493.

[3] J.G. Bennett and D.J. Murphy, Sit-ups and push-ups only-are we heading for muscular imbalance? Journal of Physical Education, Recreation \& Dance 66 (1995), 67-72.

[4] P.W. Grandjean, Obesity and the Metabolic Syndrome, in: Obesity: Prevention and Treatment, CRC Press, 2012, pp.311-342

[5] K.H. Liu, Y.L. Chan, W.B. Chan, W.L. Kong, M.O. Kong and J.C.N. Chan, Sonographic measurement of mesenteric fat thickness is a good correlate with cardiovascular risk factors: Comparison with subcutaneous and preperitoneal fat thickness, magnetic resonance imaging and anthropometric indexes, International Journal of Obesity 27 (2003), 12671273.

[6] S.N. Goldberg, G.S. Gazelle and P.R. Mueller, Thermal ablation therapy for focal malignancy a unified approach to underlying principles, techniques, and diagnostic imaging guidance, American Journal of Roentgenology 174 (2000), 323-331.

[7] D.O. Mook-Kanamori, S. Holzhauer, L.M. Hollestein, B. Durmus, R. Manniesing, M. Koek, and V.W. Jaddoe, Abdominal fat in children measured by ultrasound and computed tomography. Ultrasound in medicine \& biology, $\mathbf{3 5 . 1 2}$ (2009), 1938-1946. 
[8] J.P. Ahtiainen, M. Hoffren, J.J. Hulmi, M. Pietikäinen, A.A. Mero, J. Avela and K. Häkkinen, Panoramic ultrasonography is a valid method to measure changes in skeletal muscle cross-sectional area, European Journal of Applied Physiology 108 (2010), 273-279.

[9] G. Enzi, M. Gasparo, P.R. Biondetti, D. Fiore, M. Semisa and F. Zurlo, Subcutaneous and visceral fat distribution according to sex, age, and overweight, evaluated by computed tomography, The American Journal of Clinical Nutrition 44 (1986), 739-746.

[10] H. Vidal, Gene expression in visceral and subcutaneous adipose tissues, Annals of Medicine 33 (2001), 547-555.

[11] B.L. Wajchenberg, Subcutaneous and visceral adipose tissue: their relation to the metabolic syndrome, Endocrine reviews, 21.6 (2000), 697-738.

[12] J.R. Greenfield, K. Samaras and D.J. Chisholm, Insulin resistance, intra-abdominal fat, cardiovascular risk factors, and androgens in healthy young women with type 1 diabetes mellitus, Journal of Clinical Endocrinology \& Metabolism, 87 (2002), 1036-1040.

[13] S. Guldiken, N. Tuncbilek, O.O. Okten, E. Arikan and A. Tugrul, Visceral fat thickness determined using ultrasonography is associated with anthropometric and clinical parameters of metabolic syndrome, International Journal of Clinical Practice 60 (2006), 1576-1581.

[14] E.A. Harman, D.J. Gutekunst, P.N. Frykman, M.A. Sharp, B.C. Nindl, J.A. Alemany and R.P. Mello, Prediction of simulated battlefield physical performance from field-expedient tests, Military Medicine 173 (2008), 36-41.

[15] A.C. van Breda Vriesman, H.M. Schuttevaer, E.G. Coerkamp and J.B.C.M. Puylaert, Mesenteric panniculitis: US and CT features, European Radiology 14 (2004), 2242-2248.

[16] N. Tadokoro, S. Murano, T. Nishide, R. Suzuki, S. Watanabe, H. Murayama and Y. Saito, Preperitoneal fat thickness determined by ultrasonography is correlated with coronary stenosis and lipid disorders in non-obese male subjects, International Journal of Obesity 24 (2000), 502-507.

[17] P. Björntorp, Portal adipose tissue as a generator of risk factors for cardiovascular disease and diabetes, Arteriosclerosis, Thrombosis, and Vascular Biology 10 (1990), 493-496.

[18] S.B. Koh, J.K. Park, J.H. Yoon, S.J. Chang, S.S. Oh, J.Y. Kim and J.S.H. You, Preliminary report: A serious link between adiponectin levels and metabolic syndrome in a Korean nondiabetic population, Metabolism 59 (2010), 333337. 